\title{
Case of Acute Onset Postpartum Paraplegia: Spontaneous Spinal Epidural Hematoma-A Rare Entity
}

\author{
Praveen Kumar Pandey, MS Ortho ${ }^{1}$ Inder Pawar, MS Ortho ${ }^{1}$ Harsimar Singh ${ }^{1}$ \\ 1 Department of Orthopaedics, ESI-PGIMSR Model Hospital, \\ Address for correspondence Maj. (Dr.) Praveen Kumar Pandey, \\ Basaidarapur, New Delhi, Delhi, India \\ Graded Specialist Orthopedics, Military Hospital, Dehradun, Garhi \\ Cantt 248003, Uttarakhand (e-mail: praveen2gir@yahoo.co.in).
} Surg J 2019;5:e39-e41.

\begin{abstract}
Study Design Present study is a case report.

Objective This study was to present a rare case of acute postpartum paraplegia due to spontaneous spinal epidural hematoma (SSEH).

Background SSEH with incidence rate of 0.1 per 100,000 per year is an extremely rare cause of sudden onset neurological deficit in postpartum patients with no predisposing factors or intrapartum factor causing SSEH resulting in paraplegia.

Material and Methods We hereby present our case of acute onset postpartum paraplegia which on magnetic resonance imaging (MRI) shows epidural hematoma around thoracic 12 to lumbar 2 ( $\mathrm{T} 12-\mathrm{L} 2$ ) vertebral regions with adjacent cord changes. MRI is the investigation of choice in such cases which helps in proper timely management of patient. We planned the patient for surgical decompression of hematoma.

Keywords

- SSEH

- postpartum

- paraplegia

- MRI

- decompression

Results Patient showed rapid reversal of neurological symptoms in postoperative period.

Conclusions Acute onset postpartum paraplegia in a healthy female with no significant past history, predisposing factors or intrapartum factors may be caused by SSEH and it should be managed on emergency basis as early and proper treatment has an excellent prognosis as seen in our case.
\end{abstract}

\section{Introduction}

Spontaneous spinal epidural hematoma (SSEH) is an extremely rare entity with incidence of 0.1 per 100,000 every year. ${ }^{1}$ This is a rare cause of rapid onset neurological deficit due to which it should be diagnosed early and managed adequately to prevent permanent neurological deficit. Common cause of SSEH includes minor trauma, coagulation abnormalities, vascular malformation, transient vascular hypertension, disc herniation, and idiopathic causes. ${ }^{2}$ In review of literature, we didn't find any case of SSEH in postpartum patient leading to acute postpartum paraplegia. We present a case of acute onset postpartum paraplegia with no predisposing factor due to SSEH involving thoracic 12 to lumbar 2 vertebral levels (T12-L2).

received

May 24, 2016

accepted after revision

March 20, 2019
DOI https://doi.org/

10.1055/s-0039-1688506. ISSN 2378-5128.

\section{Case Report}

A 29-year-old female patient was referred to our department with acute onset postpartum paraplegia with bowel bladder involvement after delivery of her third child by normal vaginal delivery. Her antenatal course had no significant history with any history of antepartum low-back pain, trauma, drug use, or any physical exertion. On examination, she was conscious, well oriented, and afebrile. Neurological examination showed complete paraplegia (power 0/5 with decreased tone), associated bowel bladder involvement, and absent deep tendon reflexes at knee and ankle joint with complete sensory deficit below the level of L1. All necessary blood investigations including coagulation profile came out to be normal. Patient was planned for magnetic resonance

Copyright $\odot 2019$ by Thieme Medical Publishers, Inc., 333 Seventh Avenue, New York, NY 10001, USA. Tel: +1(212) 584-4662.
License terms

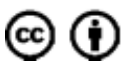


imaging (MRI) of dorsolumbar spine with screening whole spine, which revealed extradural broad based ellipsoid lesion, measuring $50 \times 9 \mathrm{~mm}$ along T12 to L2 vertebra displacing the dura anteriorly and $\mathrm{T} 1$ hyperintensity (-Fig. 1), T2 hypointensity with minimal postcontrast enhancement suggestive of early subacute bleed causing mass effect on adjacent cord with areas of altered signal intensity at the level of lesion.

On the basis of clinical evaluation and MRI report, the patient was planned for urgent decompression in emergency operation theater. After preoperative marking of D12-L2 vertebral levels under fluoroscopic guidance, midline exposure done and paraspinal muscles elevated upto medial edge of transverse processes on both sides with the help of cobb's and broad osteotome. Intraoperatively, bilateral complete laminectomy of L1 vertebrae was done with removal of ligamentum flavum, a well-defined firm organized hematoma dark reddish black in color $(5 \times 1 \mathrm{~cm})$ was seen over the dura which was removed (-Fig. 2) and checked for adequate decompression utilizing infant feeding tube. Sample removed was sent for histopathological examination reported as organized hematoma. Postoperatively, patient recovered rapidly in terms of neurological deficit and was discharged on 10th postoperative day with almost complete neurological recovery and improved bladder bowel incontinence. At the time of discharge (10th postoperative day), patient regained power of $4 / 5$ from $0 / 5$ preoperatively, improved tone, sensory improvement by $80 \%$, and regained bowel bladder control. At last follow-up of 2 years, patient recovered completely in terms of functional and neurological

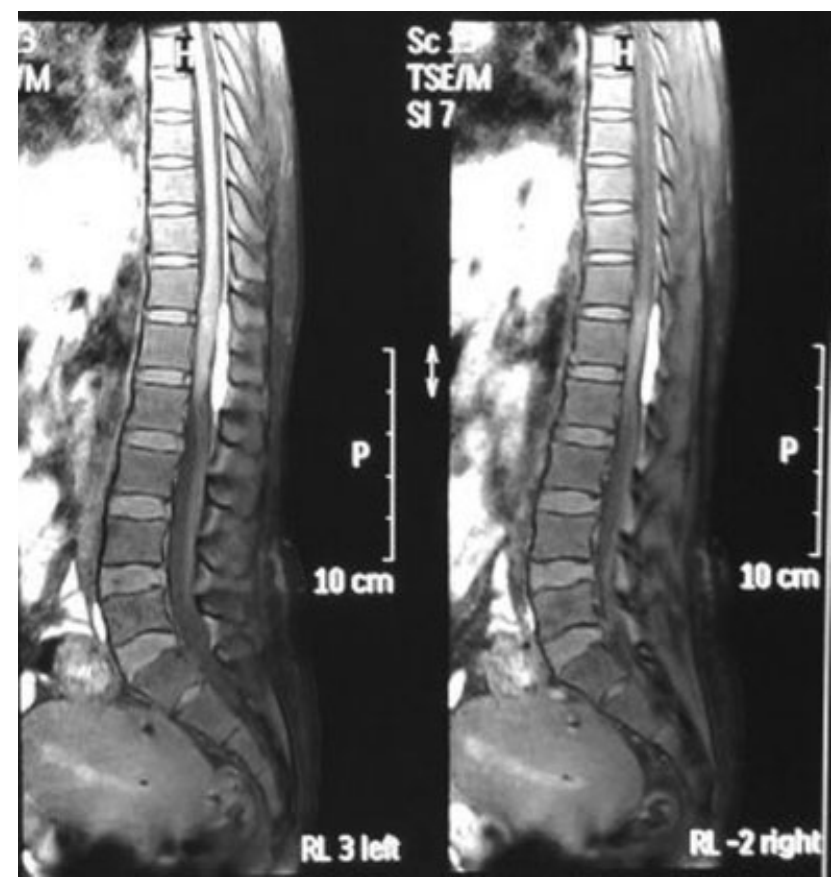

Fig. 1 MRI spine sagittal cuts showing extradural broad based ellipsoid lesion measuring $50 \times 9 \mathrm{~mm}$ along T12-L2 vertebra displacing the dura anteriorly and T1 hyperintensity. L, lumbar; MRI, magnetic resonance imaging; $\mathrm{RL}$, right to left; sc, section; sl, sliceo; $T$, thoracic; tse, turbo spin echo.

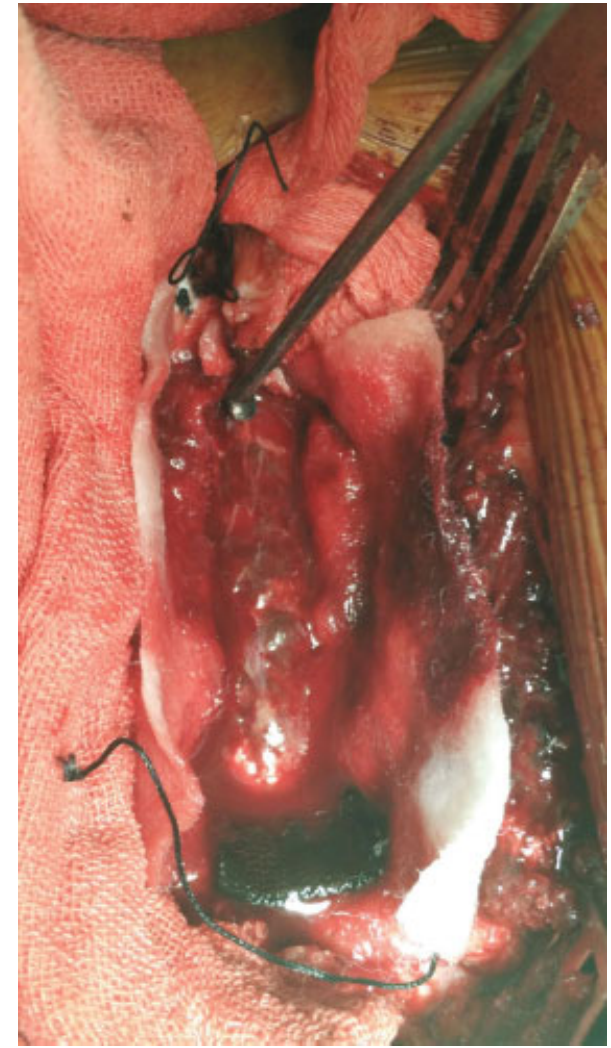

Fig. 2 Intraopertive picture after L1 laminectomy showing welldefined firm organized hematoma over the dura which was removed. L, lumbar.

evaluation. Patient was walking normally with normal bowel bladder control at the last follow-up.

\section{Discussion}

In 1869 , first case of SSEH was reported by Jackson (reported by Fedor et $\mathrm{al}^{3}$ ). Over 400 cases have been reported in the world literature with largest review of 107 patients of SSEH by Fedor et al. $^{3}$ This is more common in adult males with M:F ratio of $1.4: 1^{4}$ and commonly involves cervical-thoracic region.

Acute postpartum paraplegia commonly occurs due to predisposing factors, that is, trauma, coagulation abnormalities, vascular malformations, preeclampsia or iatrogenic, due to spinal needle for anesthesia during labor. When acute postpartum paraplegia occurs due to SSEH without any predisposing factors mentioned above, as seen in our case, is an extremely rare occurrence and not reported earlier in literature. SSEH in relation to pregnancy have been reported very few times in literature. ${ }^{5}$ SSEH originates from venous blood or arterial blood is still a controversial issue with more inclination in literature toward acceptance of venous blood collection and resultant hematoma formation. ${ }^{2,6,7}$ During pregnancy, hormonal changes may increase the fragility of vessel wall causing SSEH and may be the cause of acute postpartum paraplegia in our case. ${ }^{8}$

In cases with acute postpartum paraplegia with bowel bladder involvement, SSHE should always be kept in mind as a cause of acute onset postpartum paraplegia. Other 
differentials to be included are epidural abscess, acute prolapsed intervertebral disc (PIVD), acute spinal ischemia, spondylitis, transverse myelitis, and dissecting aortic aneurysm. ${ }^{9}$

MRI is the best modality to evaluate and diagnose SSEH. It helps in early diagnosis, accurate determination of haematoma size, level of involvement, adjacent spinal cord changes, and amount of cord compression so that patient can be managed in time and properly with surgical intervention if required $^{2}$ as soon as possible to improve the chances of neurological recovery. ${ }^{10}$ But MRI characteristics which can predict the outcome of management don't exist in literature at present. ${ }^{11}$

Commonly done procedure for SSEH includes complete laminectomy with hematoma decompression. We have done the same in our patient with excellent follow-up result. When SSEH occurs due to coagulation abnormalities or associated with mild neurological deficit or neurological deficit improving on its own, such cases can be managed conservatively with success. ${ }^{12}$ In review of literature, we found that postoperative recovery after SSEH depends on preoperative neurological deficit and time between symptom onset and surgery. ${ }^{13-15}$ Better prognosis found in cases associated with initial less severe neurological deficit and with shorter time to surgery from the onset of symptom. ${ }^{13-16}$ We found excellent result in our patient as we have operated our patient within 48 hours from onset of symptoms.

\section{Conclusion}

Acute onset postpartum paraplegia in a healthy female with no significant past history, predisposing factors or intrapartum factors may be caused by SSEH which is an extremely rare pathology during pregnancy/postpartum. Such cases must be managed on emergency basis as early and proper treatment has an excellent prognosis.

Conflicts of Interest

None declared.

\section{References}

1 Holtås S, Heiling M, Lönntoft M. Spontaneous spinal epidural hematoma: findings at MR imaging and clinical correlation. Radiology 1996;199(02):409-413

2 Baek BS, Hur JW, Kwon KY, Lee HK. Spontaneous spinal epidural hematoma. J Korean Neurosurg Soc 2008;44(01):40-42

3 Fedor M, Kim ES, Ding K, Muizelaar JP, Kim KD. Spontaneous spinal epidural hematoma: a retrospective study on prognostic factors and review of the literature. Korean J Spine 2011;8(04):272-282

4 Chen CL, Lu CH, Chen NF. Spontaneous spinal epidural haematoma presenting with quadriplegia after sit-ups exercise. Am J Emerg Med 2009;27(09):1170.e3-1170.e7

5 Singh H, Aggarwal S, Gupta R, Yadav RK. Spontaneous spinal epidural haemorrhage. an unusual cause of paraplegia. JIACM 2008;9(02):136-139

6 Beatty RM, Winston KR. Spontaneous cervical epidural hematoma. A consideration of etiology. J Neurosurg 1984;61(01):143-148

7 Farias JP, Lima JA, Antunes JL. Subacute cervical epidural hematomas. Surg Neurol 1994;42(05):414-416

8 Fujimaki H, Nakazawa T, Ueno M, et al. Spinal subarachnoid hematoma in a woman with HELLP syndrome: a case report. J Med Case Reports 2012;6:152

9 Taniguchi LU, Pahl FH, Lúcio JED, et al. Complete motor recovery after acute paraparesis caused by spontaneous spinal epidural hematoma: case report. BMC Emerg Med 2011;11:10

10 Oumar C, Habibou L, Régis OT, et al. Spontaneous spinal epidural haematoma causing paraplegia: a case report. World J Neurosci 2015;5(04):270-274

11 Hsieh CT, Chang CF, Lin EY, Tsai TH, Chiang YH, Ju DT. Spontaneous spinal epidural hematomas of cervical spine: report of 4 cases and literature review. Am J Emerg Med 2006;24(06):736-740

12 Fukui MB, Swarnkar AS, Williams RL. Acute spontaneous spinal epidural hematomas. Am J Neuroradiol 1999;20(07):1365-1372

13 Kiehna EN, Waldron PE, Jane JA. Conservative management of an acute spontaneous holocord epidural hemorrhage in a hemophiliac infant. J Neurosurg Pediatr 2010;6(01):43-48

14 Muthukumar N. Chronic spontaneous spinal epidural hematoma - a rare cause of cervical myelopathy. Eur Spine J 2003;12(01):100-103

15 Sakakibara R, Yamazaki M, Mannouji C, et al. Urinary retention without tetraparesis as a sequel to spontaneous spinal epidural hematoma. Intern Med 2008;47(07):655-657

16 Liu WH, Hsieh CT, Chiang YH, Chen GJ. Spontaneous spinal epidural hematoma of thoracic spine: a rare case report and review of literature. Am J Emerg Med 2008;26(03):384.e1-384.e2 